\title{
Editorial
}

\section{Virtual Ophthalmology Rotations-A Real Possibility during the COVID-19 Pandemic and Beyond}

\author{
Edmund Tsui, MD ${ }^{1, *}$ Michael B. Wells, MD²,* Mubarik Mohamed, BS ${ }^{2} \quad$ Christian M. Felix, BA ${ }^{1}$ \\ JoAnn A. Giaconi, MD ${ }^{1}$ \\ 1 UCLA Stein Eye Institute, David Geffen School of Medicine at UCLA, \\ Los Angeles, California \\ 2 The Ohio State University Department of Ophthalmology and Visual \\ Science, Havener Eye Institute, Columbus, Ohio \\ J Acad Ophthalmol 2020;12:e195-e199.
}

As the coronavirus disease-2019 (COVID-19) pandemic spreads worldwide and throughout the United States, medical education has been challenged to create alternative clinical experiences amidst a dynamic enviroment. ${ }^{1}$ Medical schools temporarily suspended students from direct patient care to help reduce the spread of severe acute respiratory syndrome coronavirus 2 (SARS-CoV-2) and preserve health systems' personal protective equipment, and transitioned teaching to online platforms wherever possible. In response, the Coalition on Physician Accountability listed recommendations on May 11, 2020 regarding the 2020-2021 residency cycle. ${ }^{2}$ Briefly, in-person away rotations are highly discouraged or even prohibited by institutions, except in limited circumstances; digital interviews and virtual tours will replace the traditional residency interview visits.

Given differing regional infection rates and resource allocations, potential disparities in clinical rotation experiences may ensue. Ophthalmology programs can mitigate these inequities by creating robust virtual experiences that allow students to learn ophthalmic pathology and treatments, hone some of their clinical skills, and explore residency curriculum and culture while maintaining the safety of students, educators, and patients. Currently, specialty-specific guidelines are posted on the Association of American Medical Colleges Web site, including those of the Association of University Professors of Ophthalmology (AUPO), whereby clinical departments are in the process of operationalizing virtual remote rotations with limited offerings through Visiting Student Learning Opportunities. ${ }^{3}$ Specifically, the AUPO has discouraged in-person away rotations given ongoing travel-related concerns, except in specific circumstances. ${ }^{3,4}$ The 2020-2021 match timeline has already been affected by cancellations of planned student rotations, which will likely persist throughout the upcoming academic year. Visiting subinternships are crucial for students applying into competitive specialties, such as ophthalmology, in providing advanced clinical experiences and the chance to learn about other programs. ${ }^{5}$

Co-first authors.
Therefore, innovative teaching approaches are needed to ensure students have access to clinical rotations while ensuring the safety of patients, staff, and trainees. In this environment, medical schools have increasingly incorporated virtual learning as a medium to provide instruction to students. Challenges exist in incorporating this virtual medium of learning to both the preclerkship and clerkship learning environments. We offer our perspective from both the UCLA Stein Eye Institute and the Ohio State University (OSU) Department of Ophthalmology and Visual Science on virtual rotations and detail our pilot experiences using virtual examinations.

\section{Overview of Virtual Ophthalmology Rotations}

The OSU Department of Ophthalmology has developed and implemented a virtual ophthalmology shadowing clerkship elective for 4 th year medical students. The virtual shadowing experience is composed of four sections: patient workup with technicians/imaging specialists, patient interview and general exam with attending ophthalmologist, the slit lamp exam with attending ophthalmologist, and didactics. Using a smartphone, a videoconferencing app such as FaceTime, and a simple tabletop stand, the medical student was present to observe all aspects of the patient encounter. Patients were consented for telehealth visits to use videoconferencing software for educational purposes.

\section{Patient Workup}

A departmental iPhone was carried room to room and placed on the tabletop stand during each aspect of the patient workup. Through this virtual format, medical students had the opportunity to experience the complete workup of an ophthalmology patient and observe the role of the ophthalmic technician and imaging specialist in the care of patients. Students were able to observe visual acuity testing, refraction, tonometry via Tono-Pen and Goldmann applanation, and the initial history of present illness gathered by the technician.

Copyright $\odot 2020$ by Thieme Medical Publishers, Inc., 333 Seventh Avenue, New York, NY 10001, USA.

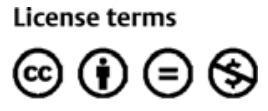

Edmund Tsui, MD, UCLA Stein Eye 10.1055/s-0040-1716714. Institute, 100 Stein Plaza, Los ISSN 2475-4757.

Angeles, CA 90095

(e-mail: ETsui@mednet.ucla.edu).
Tel: $+1(212) 760-0888$. 
Students were also able to observe the process of obtaining ophthalmic imaging, including optical coherence tomography and color fundus photography.

\section{Patient Interview and Procedures}

The student was able to observe the complete interview between the patient and attending ophthalmologist. Additionally, they were able to observe a variety of patient populations within comprehensive ophthalmology and the subspecialties of medical and surgical retina, glaucoma, and cornea. They were also able to observe in-office procedures such as intravitreal injections and corneal suture removal.

\section{Virtual Slit Lamp Examinations}

Virtual slit lamp examinations were piloted at both UCLA Stein Eye Institute and The Ohio State Havener Eye Institute. The student observed the detailed ophthalmic physical exam through use of the teaching scope and a slit lamp adapter that placed the iPhone's rear-facing camera on the slit lamp eyepiece or on an observer scope. To connect the student, who was at a remote location, and the ophthalmologist in the clinic, we used either Zoom (San Jose, CA) teleconferencing software or FaceTime (Cupertino, CA) on iPhones. The student was able to appreciate exam findings and hear a narration of those findings by the attending physician in real-time.

\section{Didactics}

As part of the OSU virtual rotation, students were scheduled for 1 half-day each week to review ophthalmology cases (27 total) with the medical student clerkship director via Zoom. Students were given these cases prior to the session, and presented their exam findings, differential diagnoses, and diagnostic/therapeutic interventions to the attending physician. Through this experience, students had the opportunity to build their clinical reasoning skills in ophthalmology and demonstrate their knowledge. Students also participated in all virtual department Grand Rounds and were invited to weekly evening resident lectures.

\section{Hardware}

Slit lamp adapters were purchased from Digital Eye Center (https://www.digitaleyecenter.com/product/universalslit-lamp-adapter-smartphone-tablet/). These are universal slit lamp adapters that fit most slit lamp eyepieces and brands of smartphones (-Fig. 1). Adapters can be placed on the main slit lamp eyepiece or on the observer scope eyepiece of a Haag-Streit BQ-900 slit lamps (-Fig. 2).

\section{Slit Lamp Illumination}

Anterior slit lamp examinations were easily performed and had high-quality images on both the iPhone and desktop (-Fig. 3). However, for the student observer video of the posterior segment examination initially was washed out and too bright to see details. Reducing light to the lowest intensity and increasing magnification appeared to alleviate some of these issues; however, this was time consuming for the examiner. To alleviate this problem, neutral density filters were placed in the tube of the teaching scope ocular as a trial and we found that a filter with optical density of 1.5 was best (https://www.edmundoptics.com/p/15-od-25mm-diameter-reflective-nd-filter/1935/) with good viewing results for both anterior and posterior examinations. A representative video examination demonstrating anterior and posterior segment examinations is available online ( - Video $\mathbf{1}$ ).

\section{Video 1}

Video of a clinical encounter demonstrating anterior segment examination of a pseudophakic patient and posterior segment examination including view of the optic disc at different magnifications. Online content including video sequences viewable at: https://www. thieme-connect.com/products/ejournals/html/ 10.1055/s-0040-1716714.

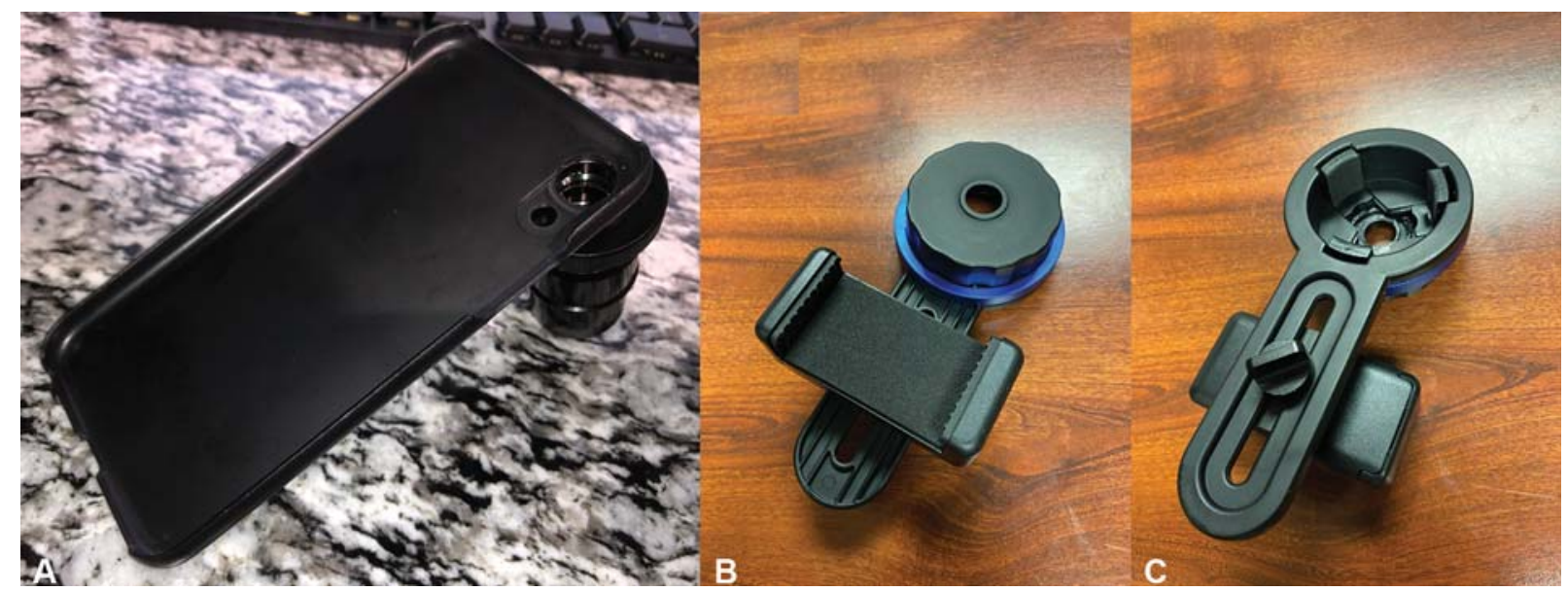

Fig. 1 Two different slit lamp adapters (A, for iPhone 10) and a universal slit lamp adapter demonstrating adjustable smartphone holder (B) and eyepiece adapter (C). 


\section{Teaching Scope Positioning}

A benefit of placing the adapter on the observer scope is that one can examine the patient without obstruction from the adapter. However, if a breath shield is located anteriorly (e.g., on the main oculars), this leaves the smartphone unprotected and potentially in need of sanitization between encounters. Breath shields may be placed in variable positions on a slit lamp, but an anterior location may be more effective for protection than posteriorly placed shields. ${ }^{6}$ Additionally, one could also consider using inclined eyepiece adapters that extend the teaching scope through the slit lamp shields (-Fig. 2B).

\section{Limitations of Virtual Slit Lamp Examinations}

There are several limitations to using teleconferencing software for clinical rotations. Neither Zoom nor FaceTime have manual focusing capabilities, so dynamic adjustment of the slit lamp may be needed to focus the image for the student observer. Another inherent limiting factor of virtual examination is the requirement of smartphones and/or a laptop or desktop computer. Not all students have access to iPhones and thus use of Android operating system or other smartphones will have to be explored. Alternatively, students may use a computer that is located at the medical school or hospital campus to view the exams or a personal encrypted

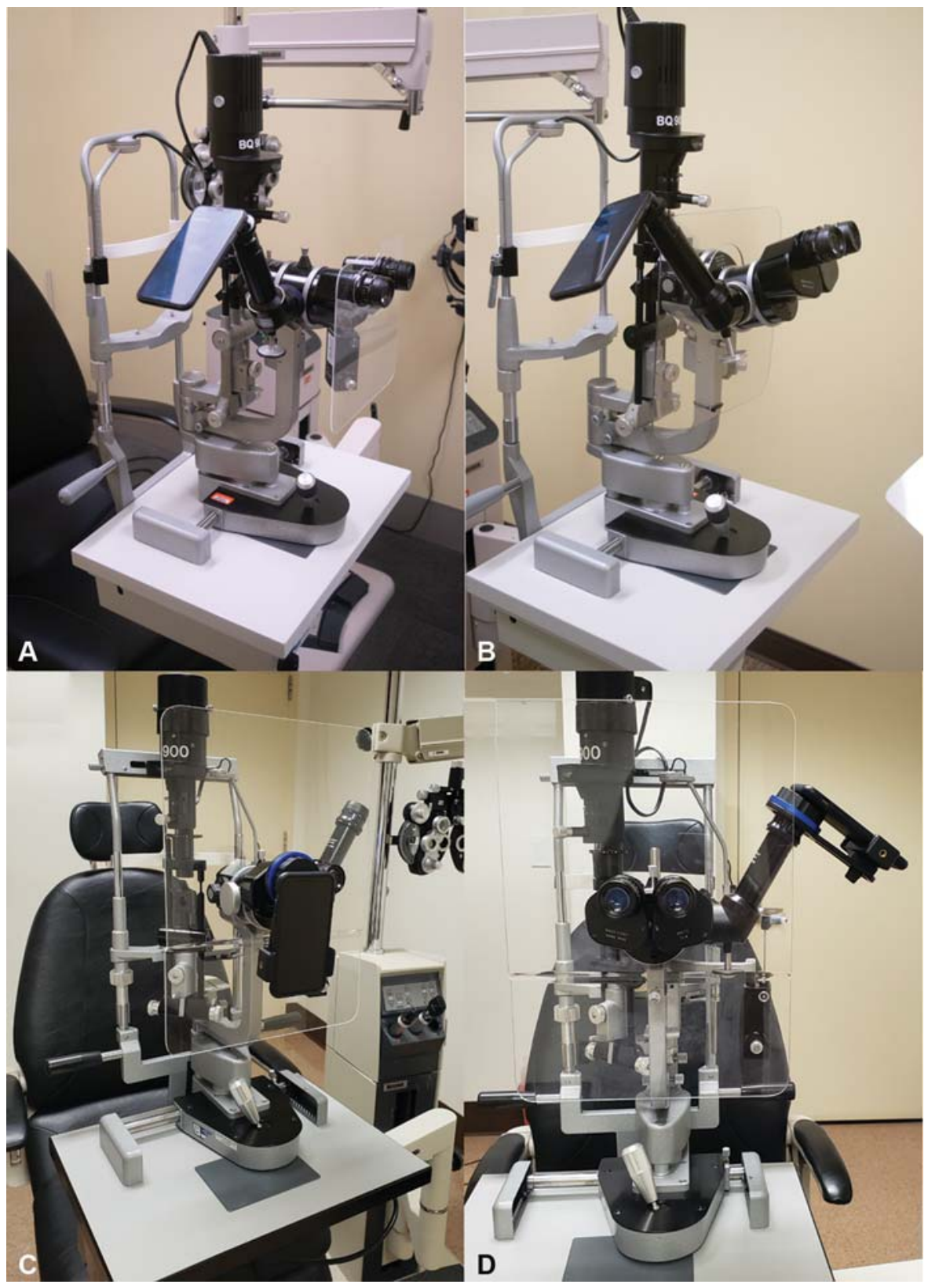

Fig. 2 Clinical set up of a slit lamp adapter on a slit lamp (Haag Streit BQ-900) with a breath shield and an anteriorly located shield (A) and another example of a posteriorly located breath shield with an inclined eyepiece adaptor that extended the slit lamp and observer scope through the opening in the breath shield (B). A second set-up with an anteriorly located larger breath shield houses a universal slit lamp on the main eyepiece (C) and also on the observer scope (D). 

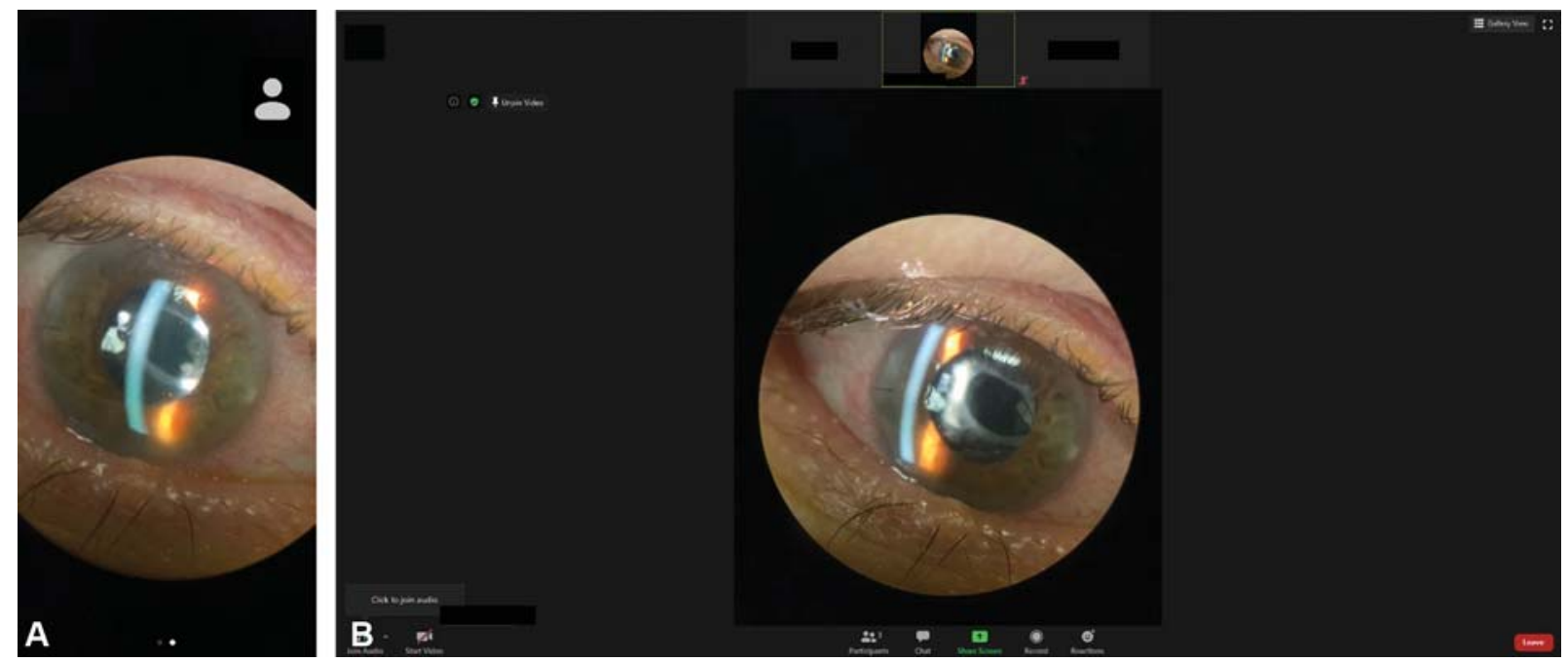

Fig. 3 Virtual slit lamp examination images using Zoom on iPhone (A) and desktop (B) showing a slit lamp image of a patient with uveitis with keratic precipitates $(\mathrm{A}$, arrow).

computer. Other platforms for videoconferencing were not tested, but could be considered, such as Microsoft Teams, WebEx, Skype, or Google Hangouts. Of note, health care organizations may vary on what teleconferencing software is permitted. In general, a secure Internet connection should be used in addition to obtaining patient consent. During the pandemic, the Office for Civil Rights at the U.S. Department of Health and Human Services has permitted use of non-Health Insurance Portability and Accountability Act (HIPAA)-compliant video chat applications, such as FaceTime, without potential penalty when providing care in good faith. ${ }^{7}$ However, this is a temporary policy, likely meaning that users will need to revert to HIPAA-compliant applications in the future. Examples of these include Zoom for Healthcare, Microsoft Teams, or WebEx. Therefore, it is anticipated that our use of FaceTime for a virtual rotation will transition to a HIPAAcompliant application, such as Microsoft Teams, in the future.

Other limitations of virtual rotations are the lack of inperson exposure to ophthalmic surgery. Surgical videos can be recorded, as is traditionally done, and made available online for trainees to watch asynchronously. Alternatively, it may be possible to use similar technology as described above in the operating room to allow for synchronous remote viewing by a medical student.

\section{Long-Term Implications of Virtual Rotations and Examinations}

Health care workers may be at an increased risk from exposure to SARS-CoV-2 in the workplace, and in particular, ophthalmologists may be at a higher risk given close face-toface examinations, ${ }^{8}$ therefore it is important to reduce risk of transmission as much as possible. As the pandemic evolves and some medical students in the United States begin to return to more normal day-to-day rotations, we hope that our experiences offer other programs insight into how virtual exams may be utilized to continue providing safe clinical exposure during the COVID-19 pandemic, especially for students still unable to participate in visiting away rotations. Feedback from rotating students has been positive with one student commenting: "As a student, my experience during the virtual ophthalmology clerkship was overwhelming positive. As my first ophthalmology rotation of medical school, this experience provided sufficient exposure to help me make a career decision to pursue this specialty. I was able to see findings in real time with the attending, ask questions about what I was seeing, and have those findings pointed out and explained to me. In terms of mentorship and connecting with faculty I did not feel the virtual form inhibited me from forming these connections."

Lastly, the use of virtual slit lamp examinations provides a unique opportunity to allow students (or other providers) to view these clinical encounters, which could be useful for teaching rounds or multidisciplinary conferences. Since these slit lamp adapters are considerably more affordable than formal slit lamp cameras, this may lead to a wider availability of video or photographs of clinical findings for teaching purposes. Ultimately, virtual rotations in the future may also offer medical students opportunities that may have been inaccessible before due to the costs of travel, accommodation, and scheduling.

\section{Funding}

This work is supported by an unrestricted grant from Research to Prevent Blindness, Inc. to the Department of Ophthalmology at UCLA.

Conflict of Interest

None declared.

\section{Acknowledgments}

The authors thank E. Mitchel Opremcak, MD, Clinical Professor, OSU Department of Ophthalmology and Visual Science for the donation of additional teaching scopes and inclined eyepiece adapters. 


\section{References}

1 Wong TY, Bandello F. Academic ophthalmology during and after the COVID-19 pandemic. Ophthalmology 2020;127(08):e51-e52

2 Final Report and Recommendations for Medical Education Institutions of LCME-Accredited, U.S. Osteopathic, and Non-U.S. Medical School Applicants. Association of American Medical Colleges. Available at: https://www.aamc.org/system/files/2020-05/covid19_Final_Recommendations_05112020.pdf. Accessed July 5, 2020

3 Specialty Response to COVID-19. Association of American Medical Colleges. Available at: https://students-residents.aamc.org/ applying-residency/article/specialty-response-covid-19/. Accessed July 5, 2020

4 Quillen DA, Siatkowski RM, Feldon S. Association of University Professors of Ophthalmology. COVID-19 and the ophthalmology match. Ophthalmology 2020. S0161-6420(20)30641-2
5 Patel SB, Kelly LD. Visiting student away rotations in ophthalmology: a study of medical students' experiences and perspectives. J Acad Ophthalmol 2020;12:52-56

6 Liu J, Wang AY, Ing EB. Efficacy of slit lamp breath shields. Am J Ophthalmol 2020:S0002-9394(20)30234-8

7 Office for Civil Rights. Notification of Enforcement Discretion for Telehealth Remote Communications during the COVID-19. Nationwide Public Health Emergency. Department of Health and Human Services. Available at: https://www.hhs.gov/hipaa/forprofessionals/special-topics/emergency-preparedness/notification-enforcement-discretion-telehealth/index.html Accessed August 4, 2020

8 Kuo IC, O'Brien TP. COVID-19 and ophthalmology: an underappreciated occupational hazard. Infect Control Hosp Epidemiol 2020:1-2 